OPEN ACCESS

Edited by:

Remi N. Charrel,

Aix Marseille Université, France

Reviewed by:

Artur Summerfield,

Institute of Virology and Immunology

(IVI), Switzerland

Amy Schuh,

Centers for Disease Control and

Prevention (CDC), United States

${ }^{*}$ Correspondence:

Dana L. Vanlandingham dlvanlan@vet.k-state.edu

Specialty section:

This article was submitted to Emerging and Reemerging Viruses,

a section of the journal

Frontiers in Virology

Received: 11 June 2021

Accepted: 13 August 2021

Published: 17 September 2021

Citation:

Park SL, Huang Y-JS, Lyons AC,

Ayers VB, Hettenbach SM,

McVey DS, Noronha LE, Burton KR,

Hsu W-W, Higgs S and

Vanlandingham DL (2021) Mosquito

Saliva Modulates Japanese

Encephalitis Virus Infection in

Domestic Pigs. Front. Virol. 1:724016.

doi: 10.3389/fviro.2021.724016

\title{
Mosquito Saliva Modulates Japanese Encephalitis Virus Infection in Domestic Pigs
}

\begin{abstract}
So Lee Park ${ }^{1,2}$, Yan-Jang S. Huang ${ }^{1,2}$, Amy C. Lyons ${ }^{1,2}$, Victoria B. Ayers ${ }^{1,2}$, Susan M. Hettenbach ${ }^{2}$, D. Scott McVey ${ }^{1,3}$, Leela E. Noronha ${ }^{4}$, Kenneth R. Burton ${ }^{2,5}$, Wei-Wen Hsu ${ }^{6}$, Stephen Higgs ${ }^{1,2}$ and Dana L. Vanlandingham ${ }^{1,2 *}$

1 Department of Diagnostic Medicine and Pathobiology, College of Veterinary Medicine, Kansas State University, Manhattan, KS, United States, ${ }^{2}$ Biosecurity Research Institute, Kansas State University, Manhattan, KS, United States, ${ }^{3}$ School of Veterinary Medicine and Biomedical Sciences, University of Nebraska-Lincoln, Lincoln, NE, United States, ${ }^{4}$ Arthropod-Borne Animal Diseases Research Unit, Center for Grain and Animal Health Research, Agricultural Research Service, United States Department of Agriculture, Manhattan, KS, United States, ${ }^{5}$ National Agricultural Biosecurity Center, Kansas State University, Manhattan, KS, United States, ${ }^{6}$ Department of Statistics, College of Arts and Sciences, Kansas State University, Manhattan, KS, United States
\end{abstract}

Japanese encephalitis virus (JEV) is a mosquito-borne flavivirus that is the leading cause of pediatric viral encephalitis in Asia. Japanese encephalitis virus is transmitted by Culex species mosquitoes that also vector several zoonotic flaviviruses. Despite the knowledge that mosquito saliva contains molecules that may alter flavivirus pathogenesis, whether or not the deposition of viruses by infected mosquitoes has an impact on the kinetics and severity of JEV infection has not been thoroughly examined, especially in mammalian species involved in the enzootic transmission. Most JEV pathogenesis models were established using needle inoculation. Mouse models for West Nile (WNV) and dengue (DENV) viruses have shown that mosquito saliva can potentiate flavivirus infections and exacerbate disease symptoms. In this study, we determined the impact of mosquito salivary components on the pathogenesis of JEV in pigs, a species directly involved in its transmission cycle as an amplifying host. Interestingly, co-injection of JEV and salivary gland extract (SGE) collected from Culex quinquefasciatus produced milder febrile illness and shortened duration of nasal shedding but had no demonstrable impact on viremia and neuroinvasion. Our findings highlight that mosquito salivary components can differentially modulate the outcomes of flavivirus infections in amplifying hosts and in mouse models.

Keywords: mosquito, saliva, Japanese encephalitis virus, pig, pathogenesis

\section{INTRODUCTION}

Japanese encephalitis virus (JEV) is a member of the genus Flavivirus of the family Flaviviridae, which contains several mosquito-borne human pathogens, including dengue (DENV), West Nile (WNV), and yellow fever viruses (YFV) (1). Currently, JEV is the leading cause of pediatric viral encephalitis in Asia, with annual estimates of 68,000 cases with a $30 \%$ mortality rate (2-4). It is also an agricultural pathogen, capable of causing non-suppurative encephalitis in piglets (5-7) and an array of reproductive disease outcomes in mature pigs including abortions, stillbirths, and transient infertility (7-9). The virus is endemic to the Asia-Pacific region (4), but the detection 
of JEV genome has been reported in Italy $(10,11)$ and Angola (12), suggesting the possibility of dispersal and establishment of enzootic cycles in locations where amplifying hosts and competent vectors are present.

Japanese encephalitis virus infects a wide variety of vertebrate hosts in nature including cattle, horses, dogs, and reptiles (13). Avian and swine species are the principle amplifying hosts that support the enzootic transmission of JEV. Japanese encephalitis pathogenesis has been investigated in amplifying hosts and incidental hosts under laboratory conditions such as in mice (14-17), non-human primates (18-20), chickens (21), ducklings (21-23), and pigs (5, 24-27). The majority of these published studies induced neuroinvasive disease through needle inoculations, without considering the potential impact of mosquito saliva, which is known to contain components that modulate immune responses and alter outcomes of arbovirus infections in mammalian hosts (28-33).

The objective of this study was to determine the impact of mosquito salivary components on JEV infection in pigs. Using the established method of the collection and injection of mosquito salivary gland extract (SGE) to mimic the delivery of mosquito saliva when feeding (34-37), we hypothesized that the simultaneous delivery of mosquito SGE and infectious viruses might alter the pathological outcomes caused by JEV infection in pigs. Modulation of arbovirus infections by mosquito salivary components has been primarily demonstrated in laboratory mice but requires further evaluation with other animal models. Enhanced disease symptoms have been reported in mouse models that received mosquito saliva or SGE and challenged with alphaviruses $(33,34)$, bunyaviruses $(28,38)$, and flaviviruses $(35,37,39-41)$. However, observations in mouse models were not consistent with other small animal models. For example, challenge of hamsters (42) and chickens (43) with WNV and house finches with Western equine encephalitis virus (44) through the bites of infected mosquitoes had no demonstrable differences in disease severity nor infection outcomes when compared with needle injection. Our work established a model to study the modulation of JEV infections by mosquito salivary components in pigs. This is also the first study that evaluated the impact of mosquito saliva on flavivirus pathogenesis in a mammalian host which develops viremia and can support enzootic flavivirus transmission in nature. Understanding how mosquito saliva modulates flavivirus infections in mammalian amplifying hosts has significant implications because humans play a similar role in the urban transmission of DENV, YFV, and Zika virus.

In this study, the co-injection of SGE derived from Culex quinquefasciatus and JEV through the intradermal route altered the kinetics of JEV infection in 3-week-old white-line crossbreed domestic pigs. In contrast to the enhancement of diseases caused by mosquito saliva reported in mouse models, SGE reduced the severity of diseases caused by JEV infection as demonstrated by the development of milder febrile illness and shortened period of viral nasal shedding. Interestingly, the viral loads among the tissues collected from the central nervous system (CNS) did not differ significantly and no demonstrable effect on viremic titers were observed with the co-inoculation of SGE and JEV.
The findings suggest that the modulation of flavivirus infection by mosquito saliva may result in different infection outcomes depending on the vertebrate host species.

\section{MATERIALS AND METHODS}

\section{Virus and Cells}

Japanese encephalitis virus strain JE-91 was propagated using Aedes albopictus C6/36 cells maintained in Leibovitz (L-15) medium and stored at $-80^{\circ} \mathrm{C}(26,45)$. JE-91 strain (GenBank accession: GQ415355) was originally isolated from mosquitoes in Korea in 1991. It was passaged once in Vero cells and once in C6/36 cells prior to this study. The strain was selected as a representative strain for genotype $\mathrm{Ib}$ of JEV, which is the dominantly circulating genotype in the endemic region. African green monkey kidney Vero76 cells maintained in L15 media were used to determine infectious virus titers of virus stocks via median tissue culture infectious dose $\left(\right.$ TCID $\left._{50}\right)$ method $(26,46,47)$.

\section{Preparation of Mosquito Salivary Gland Extract}

Salivary glands were dissected from a colony of $C x$. quinquefasciatus $(F>30)$ originally obtained from Vero Beach, FL (48, 49). The colony tested negative for known flaviviruses using the pan-flavivirus EMF1-VD8 primer set (50). Seven- to 10-day-old female mosquitoes were surface sterilized with $70 \%$ ethanol and dissected in phosphate buffer saline (PBS) to obtain salivary glands. Fifty pairs of salivary glands were placed in approximately $1 \mathrm{ml}$ of PBS, sonicated, and centrifuged at $13,000 \mathrm{rpm}$ for $10 \mathrm{~min}$ at $4^{\circ} \mathrm{C}$ to release proteins and pellet cellular debris (51). Supernatant was collected and stored at $-80^{\circ} \mathrm{C}$ until the challenge experiment.

\section{Study Design and Sample Collection}

The study was designed to investigate the impact of mosquito salivary proteins on the tropism of JEV and tissue viral loads of experimentally challenged domestic pigs, and of disease progression. A total of 28 three-week-old white-line crossbreed domestic pigs were randomly assigned into four groups $(n=$ 4 or 10), as summarized in Table 1. Animals were co-injected with either $100 \mu \mathrm{l}$ equal volume mixtures of SGE and JEV stock containing $10^{7}$ TCID $_{50}$ of JE-91 strain (SGE+JEV, $n=10$ ) or $100 \mu \mathrm{l}$ equal volume mixtures of SGE and sterile saline (SGEonly, $n=4$ ). Other groups of animals were injected with a mixture of $50 \mu \mathrm{l}$ of sterile PBS and $50 \mu \mathrm{l}$ of JEV stock (JEVonly, $n=10$ ) or $100 \mu$ l of sterile saline only (mock, $n=4$ ) to characterize JEV pathological outcomes by needle inoculation. The dosage used in our study was comparable with the dosages described in previous studies $(5,25,26,52)$, which successfully established JEV infection in domestic pigs. All animals were intradermally inoculated through a single injection at the base of the left ear. Half of the animals in each treatment group ( $n$ $=14$ ) were sacrificed at 3 days or 28 days post-infection (DPI) for the investigation of tissue tropism and viral loads during the acute and convalescent phases of JEV infection, respectively. 
TABLE 1 | Summary of the study experimental groups.

\begin{tabular}{|c|c|c|c|c|}
\hline Group & Intradermal inoculum & Total $n$ & Necropsy at 3 DPI & Necropsy at 28 DPI \\
\hline Mock & $100 \mu$ l sterile PBS & $n=4$ & $n=2$ & $n=2$ \\
\hline JEV-only & $50 \mu \mathrm{l}$ sterile PBS $+50 \mu \mathrm{l} 10^{7} \mathrm{TCID}_{50} \mathrm{JEV}$ & $n=10$ & $n=5$ & $n=5$ \\
\hline SGE-only & $50 \mu \mathrm{l}$ SGE $+50 \mu \mathrm{l}$ sterile PBS & $n=4$ & $n=2$ & $n=2$ \\
\hline SGE+JEV & $50 \mu \mathrm{l}$ SGE $+50 \mu \mathrm{l} 10^{7} \mathrm{TCID}_{50} \mathrm{JEV}$ & $n=10$ & $n=5$ & $n=5$ \\
\hline
\end{tabular}

DPI, day post-infection; PBS, phosphate buffer saline; SGE, salivary gland extract; JEV, Japanese encephalitis virus; TCID $50,50 \%$ tissue culture infectious dose.

All experimental procedures were approved by Kansas State University's Institutional Animal Care and Use Committee.

All animals were monitored daily for clinical signs, including fever $\left(\geq 40^{\circ} \mathrm{C}\right)$, mentation changes, and neurologic abnormalities. Serum and nasal swab samples were collected to characterize the kinetics of viremia and nasal shedding, respectively, as previously described (26). Blood was obtained daily until 7 DPI and then weekly until 28 DPI. Nasal swab samples were obtained daily from alternating nares until 14 DPI and then weekly until 28 DPI and processed for analysis as previously described (26).

At 3 or 28 DPI, the animals were euthanized by intramuscular injection with xylazine $(2-3 \mathrm{mg} / \mathrm{kg}$ ) and ketamine $(10-20 \mathrm{mg} / \mathrm{kg}$ ) followed by intravenous injection of sodium pentobarbital $(390 \mathrm{mg} / \mathrm{ml})$. Approximately $5 \mathrm{~mm}^{3}$ blocks of the following CNS and lymphoid tissues were collected in $1 \mathrm{ml}$ of L-15 media for the quantification of infectious viruses and viral loads: brain (olfactory bulb, olfactory peduncle, piriform cortex, midbrain, pons, thalamus, frontal lobe, parietal lobe, temporal lobe, occipital lobe, and caudate nucleus), spinal cord, nasal epithelium, olfactory neuroepithelium, medial retropharyngeal lymph node, mesenteric lymph node, medial iliac lymph node, Peyer's patches, thymus, and tonsil. All of the samples were stored at $-80^{\circ} \mathrm{C}$ prior to analysis as described previously (26).

\section{Detection of Infectious Virus and Viral Genome}

Standard plaque assay with Vero76 cells was used to detect and quantify infectious viruses in the serum and nasal swab suspensions, as previously described $(26,53)$. Infectious viral titers were calculated in plaque forming units $(\mathrm{PFU}) / \mathrm{ml}$. Infectious viral titers in $\mathrm{PFU} / \mathrm{g}$ were also calculated for the homogenized tissue samples, as previously described (26). Tissue samples were first weighed, homogenized via the TissueLyser II system (Qiagen) for $4 \mathrm{~min}$ at $26 \mathrm{~Hz}$, and centrifuged at 10,000 $\times$ $g$ for $10 \mathrm{~min}$ prior to analysis.

A previously published and validated TaqMan one-step reverse-transcriptase quantitative polymerase chain reaction (RT-qPCR) assay was used to quantify JEV genomes in the serum, nasal swab suspensions, and tissue samples (54). Reactions were performed using the iTaq Universal Probe One-step kit (BioRad) on a CFX96 Real-Time PCR Detection System (Bio-Rad) following procedures as previously described $(26,54,55)$. Tissue samples were first weighed, homogenized via the TissueLyser II system (Qiagen) for $4 \mathrm{~min}$ at $26 \mathrm{~Hz}$, and centrifuged at $10,000 \times g$ for $10 \mathrm{~min}$. Viral RNA was extracted from the serum and nasal swab suspensions with the QIAamp viral RNA extraction kit (Qiagen). Total RNA was extracted from the homogenized tissues with Trizol LS (Invitrogen). A standard curve was constructed using serially diluted RNA extract derived from a JEV stock at $8.52 \log \mathrm{TCID}_{50} / \mathrm{ml}$. Samples with $\mathrm{Ct}$ values lower than 34 were considered positive. Results were reported as genome equivalent to $\log _{\mathrm{TCID}} / \mathrm{ml}$ (geq-TCID $50 / \mathrm{ml}$ ) or $\log \mathrm{TCID}_{50} / \mathrm{g}($ geq-TCID $50 / \mathrm{g})$.

\section{Plaque Reduction Neutralization Test}

Neutralizing antibody titers were determined by plaque reduction neutralization test, as previously described $(26,56)$. Heat inactivated serum samples were serially diluted two-fold starting at 1:10 to $1: 640$ and incubated with approximately 50 PFU of JE-91 strain for $1 \mathrm{~h}$ at $37^{\circ} \mathrm{C}$. Mixtures of antibodies and viruses were inoculated onto monolayers of Vero76 cells and overlaid with L-15 media supplemented with $1 \%$ methylcellulose. Plaques were counted after 5 days of incubation at $37^{\circ} \mathrm{C}$. Neutralizing antibody titers were calculated based on a $50 \%$ or greater reduction in plaque counts $\left(\mathrm{PRNT}_{50}\right)$.

\section{Statistical Analysis}

Viral loads in tissue samples collected from the groups of animals at 3 and 28 DPI were evaluated by non-parametric KruskalWallis tests for the four groups (mock, JEV-only, SGE-only, and SGE+JEV) and post-hoc Mann-Whitney U-tests using the SAS software (version 9.4, SAS Institute, Cary, NC). Owing to the violation of normality assumption and considering time as a factor, non-parametric Kruskal-Wallis tests and post-hoc Dunn's multiple pairwise comparison tests with Bonferroni correction [Dunn-Bonferroni test (DBT)] were performed to compare temperature, viremia levels, and nasal shedding levels between the JEV-only and SGE+JEV groups. For the differences in the duration of nasal shedding between the SGE+JEV and JEVonly groups, Student's $t$-test was used for such an evaluation. Fisher's exact tests were used to analyze the difference in ataxia incidence between the groups. The R software (version 4.1.0, The $\mathrm{R}$ Foundation) was used for data graphical display.

\section{RESULTS}

All animals were healthy and had no detectable neutralizing antibodies against JEV at the start of the study. Inoculation of co-injection of JEV and SGE or JEV alone, both led to the onset of symptoms including fever and lethargy. Clinical signs of acute infections subsided near to the defervescence stage followed by the development of abnormal gait changes indicating 


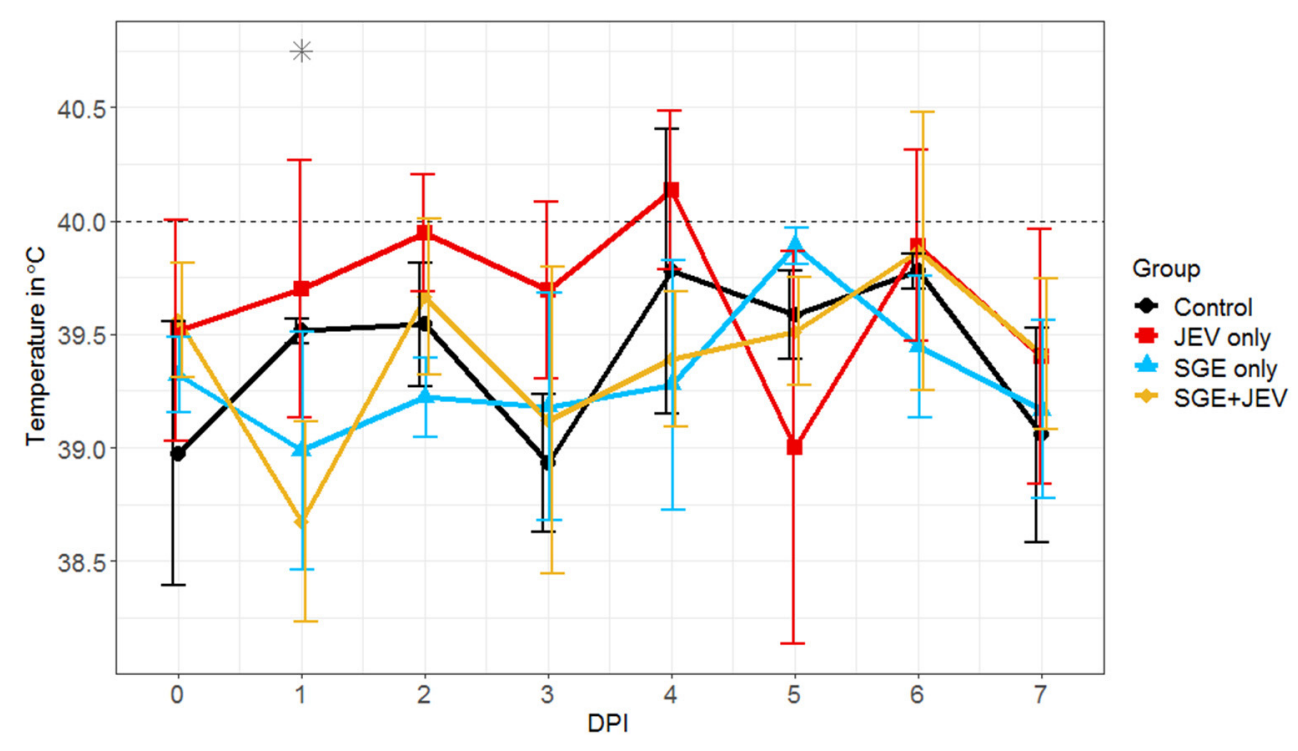

FIGURE 1 | Average body temperatures of pigs after intradermal challenge with sterile saline (control), $10^{7}$ TCID50 of JE-91 strain (JEV-only), salivary gland extract (SGE-only), and $10^{7} \mathrm{TCID}_{50}$ of JE-91 strain mixed with salivary gland extract (SGE+JEV). DPI, day post-infection. Asterisk (*) indicates the significant difference when JEV and SGE+JEV groups were compared to each other considering time as a factor and using non-parametric Kruskal-Walls test and post-hoc Dunn's multiple pairwise comparison test adjusted with Bonferroni correction.

neurological damage. The onset of clinical signs and tissue viral loads were compared to assess the impact of SGE on the kinetics and severity of JEV infection in pigs.

\section{Injection of SGE and JEV Modulated the Kinetics of Fever but Not Titers of Viremia or Nasal Shedding}

The development of fever has been consistently observed in pigs challenged with JEV $(25-27,52)$. We hypothesized that the antiinflammatory properties of mosquito saliva could potentially modulate the onset and severity of clinical diseases induced by JEV as reflected by the observed development of febrile illness $(29,33,34)$. Elevated body temperatures were detected in both SGE+JEV and JEV-only groups. One notable difference between the SGE+JEV and JEV-only groups was the time of fever onset and percentage of animals with elevated temperature (Figure 1; Supplementary Figure 1). Injection with JEV-only led to the development of fever in $10 \%$ of animals (1/10) at 1 DPI followed by the highest incidence of fever $(80 \%, 4 / 5)$ observed at 4 DPI. The animals that received the co-injection of JEV and SGE showed delayed onset of fever and did not reach the highest incidence until 6 DPI $(40 \%, 2 / 5)$. The average body temperatures of animals in the SGE+JEV group were only significantly lower than those in the JEV-only group at 1 DPI (DBT, $p=0.017$ ) but remained lower on 2, 3, and 4 DPI although the comparisons were not statistically significant (DBT, $0.155 \geq p \geq 0.999$ ). The observations suggest that the co-injection of SGE and JEV has a significant but transient impact on the dynamics of febrile illness in infected pigs.

Differential kinetics in the development of febrile illness in response to the needle inoculation of JEV and the simultaneous delivery of SGE and JEV warranted the comparison of viremic and nasal shedding profiles, two important manifestations caused by JEV infection in pigs $(26,52,57)$. All virus-challenged animals developed transient viremia, as shown in Figures 2A,B. The average viremic titers and serum viral loads among the SGE+JEV animals appeared slightly lower than the JEV-only group, but the differences were not statistically significant at any DPI (DBT, $p \geq$ 0.999). The highest average viremic titer observed in both groups were comparable to each other (SGE+JEV at 2 DPI: $2.2 \times 10^{4}$ $\pm 3.3 \times 10^{4} \mathrm{PFU} / \mathrm{ml}$; JEV-only at $3 \mathrm{DPI}: 1.1 \times 10^{5} \pm 2.9 \times 10^{5}$ $\mathrm{PFU} / \mathrm{ml}$; DBT, $p=0.396$ ) followed by the clearance of viremia at either 4 or 5 DPI. Additionally, the serum viral loads also peaked in SGE+JEV and JEV-only groups at $2\left(4.7 \times 10^{4}\right.$ geq$\left.\mathrm{TCID}_{50} / \mathrm{ml}\right)$ and $3 \mathrm{DPI}\left(4.4 \times 10^{5}\right.$ geq-TCID $\left.{ }_{50} / \mathrm{ml}\right)$, respectively. No difference was found in the serum viral loads between the two groups of animals at 2 or 3 DPI (DBT, $p \geq 0.999$ ).

Infectious viruses were isolated in nasal swabs collected from a random subset of experimentally challenged pigs (Figure 3A). Forty percent (4/10) of animals in the SGE+JEV group began to secrete infectious viruses as early as 2 DPI. Similarly, infectious virus was detected in nasal secretions collected from $60 \%(6 / 10)$ of pigs in the JEV-only group. Detection of infectious viruses persisted in both the SGE+JEV and JEV-only groups for up to 6 and 5 DPI, respectively. Detection of viral RNA demonstrated that $80 \%(8 / 10)$ of SGE+JEV pigs and $100 \%(10 / 10)$ of JEV-only pigs developed nasal shedding (Figure 3B). The duration of nasal shedding was prolonged in comparison with the durations of fever and viremia. By RT-qPCR, viral RNA was detected from 2 DPI to 7 DPI in the SGE+JEV group whereas nasal shedding was detected up to $10 \mathrm{DPI}$ in the JEV-only group. Nasal secretions had no demonstrable difference in infectious titers and viral 


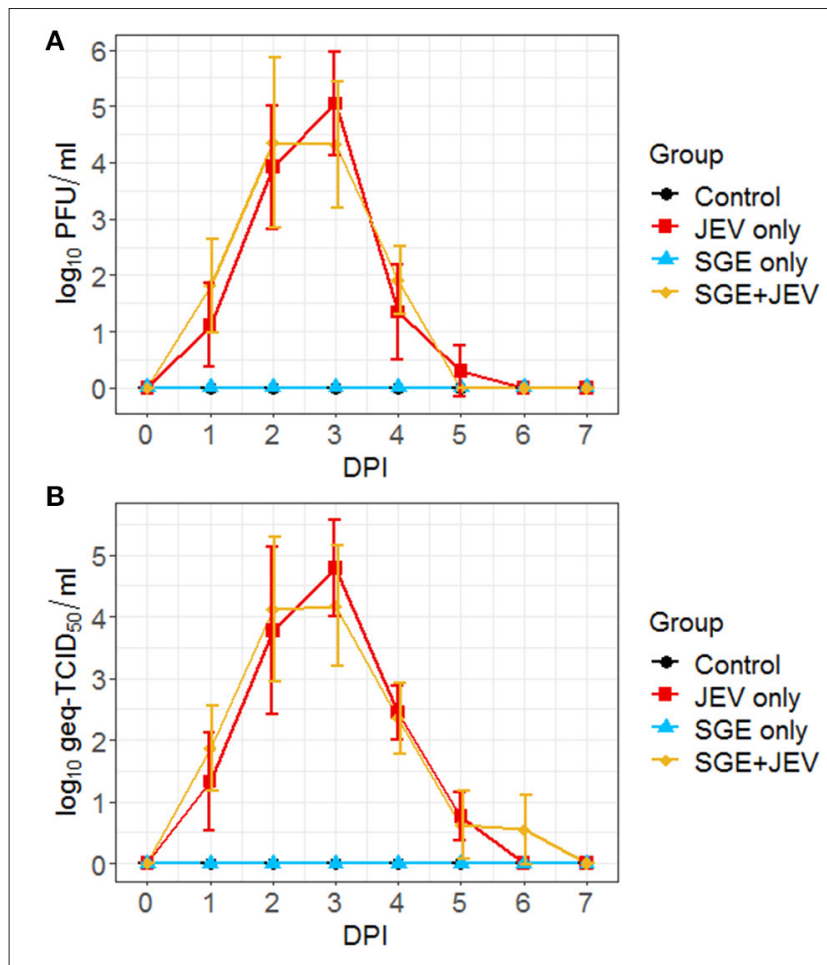

FIGURE 2 | Viremic profiles of individual animals following intradermal JEV challenge with or without SGE quantified by plaque assay (A) and RT-qPCR (B). DPI, day post-infection; PFU, plaque forming units; Geq-TCID 50 , genome equivalent-50\% tissue culture infectious dose.

RNA loads at any DPI between SGE+JEV and JEV-only groups (Kruskal-Wallis test, $p=0.113$ in infectious titer and DBT, $p$ $\geq 0.999$ for viral RNA loads). However, the average duration of nasal shedding was significantly shorter among SGE+JEV pigs $(1.8 \pm 1.3$ days $)$ than those challenged with JEV only $(3.8 \pm 1.6$ days) (one-tailed $t$-test, $t=1.925, p=0.045$ ).

The delayed onset of fever and shortened periods of nasal shedding suggest that the inclusion of SGE in the inocula altered the kinetics of acute disease signs caused by JEV infection. However, the addition of SGE did not have a demonstrable impact in quantities of infectious viruses and viral genomes in serum and nasal secretions.

\section{SGE Altered the Viral Burden in Peripheral Nervous Tissues but Had No Demonstrable Impact on the Infection Outcomes of Lymphoid and Central Nervous Tissues}

Detectable viremia led to the dispersal of JEV to lymphoid and nervous tissues through the hematogenous route in both SGE+JEV and JEV-only groups at 3 DPI (Figure 4). Consistent with the comparable viremia titers, the systemic spread of JEV was not impacted by the co-administration of SGE and JEV because the infectious titers and viral RNA loads of homogenized lymphoid tissues (peripheral lymphoid nodes, thymus, and tonsil) did not show demonstrable differences (DBT, $p \geq 0.999$ for infectious titer and Kruskal-Wallis test, $p=0.363$ for viral
RNA load) (Figure 4; Supplementary Data 1). The co-injection of JEV and SGE did, however, lead to different outcomes of JEV infection in one of the two peripheral nervous tissues examined in this study (Figure 5). Homogenized sciatic nerves obtained from SGE+JEV animals $\left(2.2 \times 10^{1} \pm 4.4 \times 10^{1} \mathrm{PFU} / \mathrm{g}\right) \mathrm{had}$ significantly lower amounts of infectious viruses than those that were injected with JEV alone $\left(2.5 \times 10^{3} \pm 4.7 \times 10^{3}\right.$ $\mathrm{PFU} / \mathrm{g}$, Mann-Whitney U test, $p=0.032$ ). However, there was no demonstrable statistical difference in the viral RNA load of the sciatic nerve samples (Mann-Whitney $U$ test, $p=0.548$ ) (Figure 4). Albeit the lack of statistical significance (MannWhitney $\mathrm{U}$ test, $0.222 \leq p \leq 0.999$ ), the average viral RNA loads in the CNS collected from the SGE+JEV group were overall lower than those from the animals that were injected with JEV alone (Figure 4).

\section{JEV Infection Caused Neurologic Signs and Persistent Infection in Pigs}

Development of trembling, paralysis, and/or ataxia of the hind limbs has been previously reported in JEV-infected swine $(5,6,26,58,59)$. Animals in the SGE+JEV and JEV-only groups had a comparable incidence of ataxia (SGE+JEV: $80 \%$ (4/5); JEV-only: 40\% (2/5); Fisher's exact test, $p=0.110$ ) (Supplementary Data 2). The development of ataxia in both groups was stochastic. In the SGE+JEV group, two animals became ataxic as early as 6 DPI and persisted until the end of the study. Two additional pigs developed bilateral hind limb ataxia: one at 11 DPI that lasted a week and the other at 25 DPI that was persistent until $28 \mathrm{DPI}$. In the JEV-only group, one pig developed gait abnormality at 15 DPI until the end of the study, while another exhibited only a 2-day period of mild rear limb ataxia between 22 and 23 DPI. Despite the apparent signs of neurologic abnormalities, all animals survived the experimental challenge and developed neutralizing antibody responses. Geometric mean $\mathrm{PRNT}_{50}$ titers were similar between the SGE+JEV (105.6) and JEV-only (91.9) groups (Mann-Whitney U test, $p=0.841$ ).

Neurological signs observed during the convalescent phase of JEV infection were consistent with the presence of viral RNA in CNS and lymphoid tissues in both groups of animals. Viral RNA was detected in at least one CNS tissue in two animals in the SGE+JEV group and one animal in the JEV-only group at 28 DPI (Figure 6). In addition to the detection of viral RNA in CNS tissues at $28 \mathrm{DPI}$, thymus and tonsil were potential sites of persistent infection. Viral loads in the thymus collected from infected pigs ranged between $3.6 \times 10^{1}$ and $8.12 \times 10^{1}$ geq$\mathrm{TID}_{50} / \mathrm{g}$ in the SGE+JEV group $(n=3)$ and between $3.85 \times 10^{1}$ and $4.23 \times 10^{1}$ geq-TCID $50 / g$ in the JEV-only group $(n=2)$. Tonsils had a higher level of viral loads as shown with average viral titers of $5.8 \times 10^{3} \pm 2.6 \times 10^{3}$ geq-TCID $_{50} / g$ from two SGE+JEV pigs and $1.6 \times 10^{3} \pm 1.4 \times 10^{3}$ geq-TCID $_{50} / g$ from three JEV-only pigs. However, there was no statistical difference across tissue samples collected at 28 DPI between the groups (Kruskal-Wallis test, $p=0.378$ ), suggesting that SGE had no impact on the persistence of JEV infection in pigs.

In summary, the impact of mosquito salivary proteins on the kinetics and severity of diseases was limited to the acute phase of 

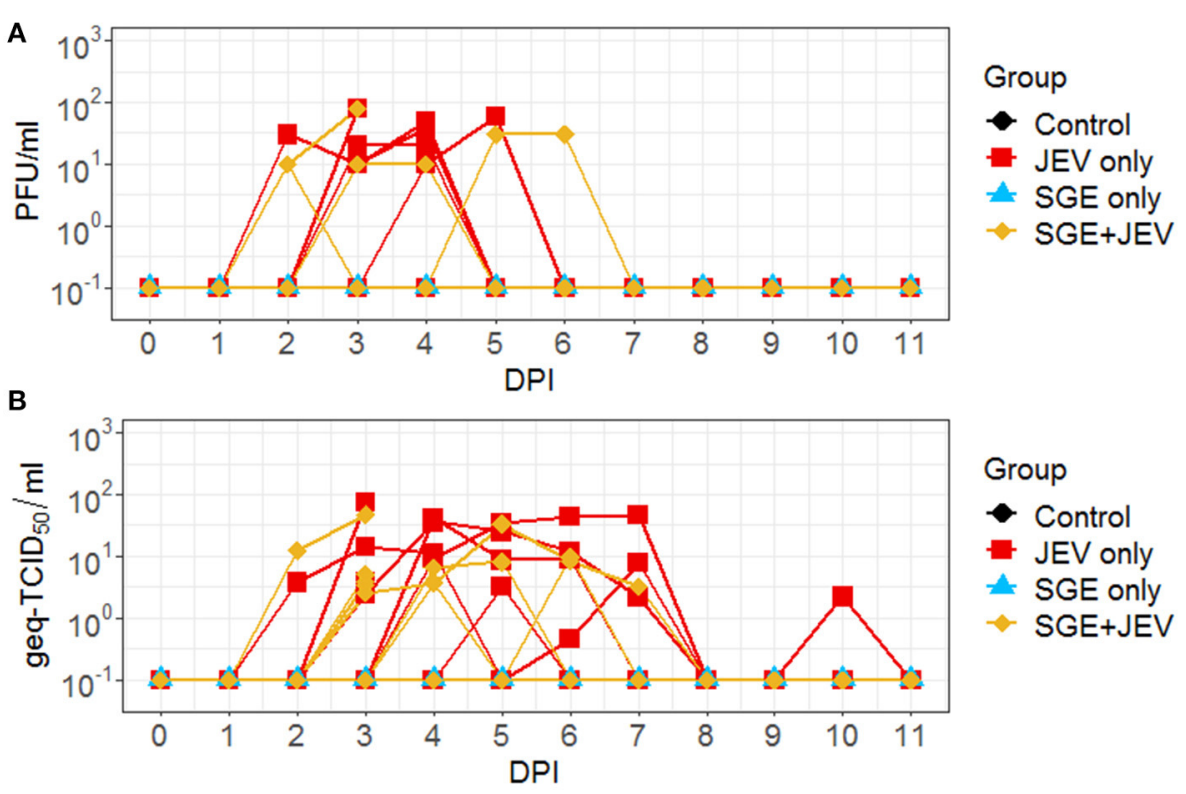

Group

- Control

- JEV only

A SGE only

$\rightarrow$ SGE+JEV

FIGURE 3 | Nasal shedding kinetics of individual animals following intradermal JEV challenge with or without SGE quantified by plaque assay (A) and RT-qPCR (B). $\mathrm{PFU}$, plaque forming units; DPI, day post-infection; Geq-TCID 50 , genome equivalent-50\% tissue culture infectious dose.
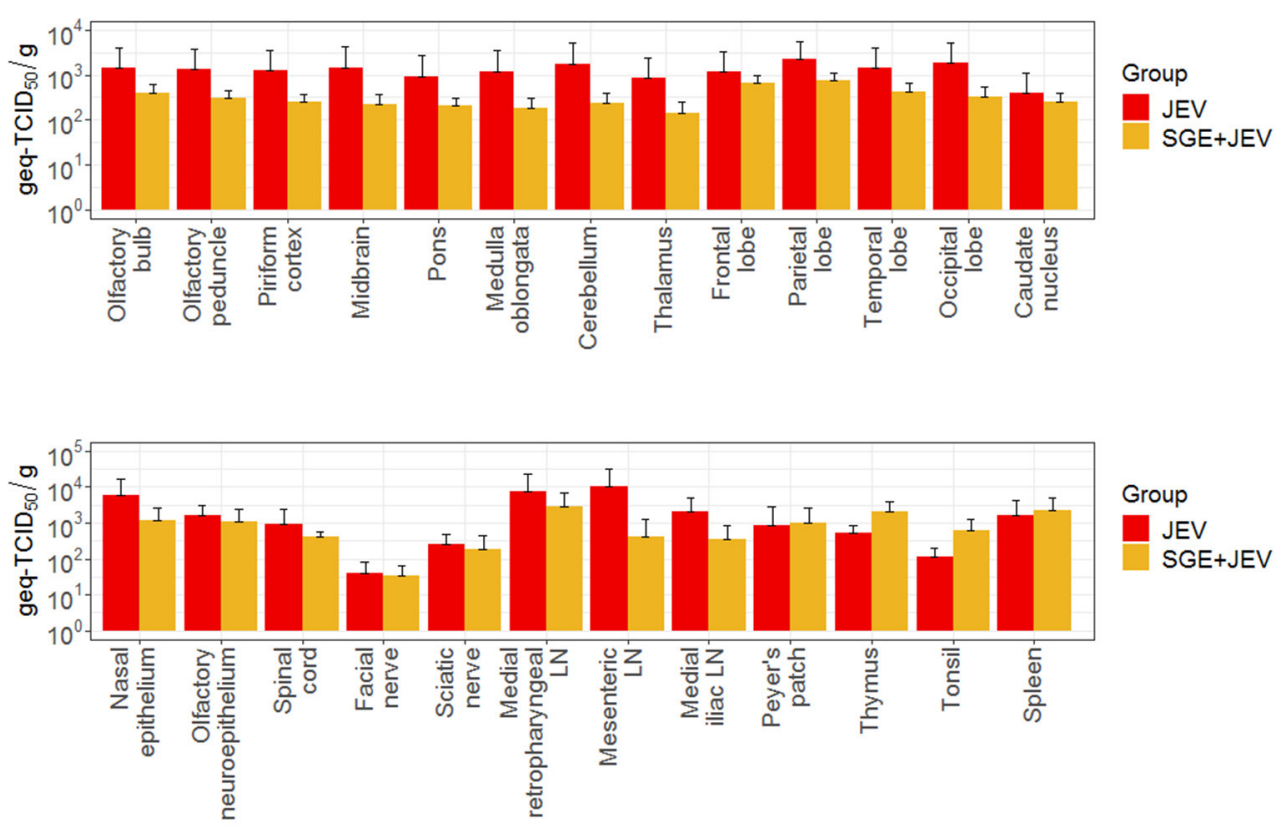

FIGURE 4 | Average viral loads of several tissues collected at three DPI following intradermal JEV challenge with or without SGE. DPI, days post-infection; Geq-TCID 50 , genome equivalent-50\% tissue culture infectious dose.

JEV infection. In comparison with pigs inoculated with JEV only, the co-injection of SGE with JEV led to milder disease based on the delayed onset of fever, shortened nasal shedding, and reduced CNS viral loads. However, the reduced severity of acute JEV infection in the SGE+JEV group had no demonstrable impact on the frequency of neurological diseases and persistent infection.

\section{DISCUSSION}

Mosquitoes play an integral role in the transmission of arboviruses. Mosquito salivary components have been increasingly recognized as an important factor that modulates vertebrate immune responses and, as a consequence, disease 

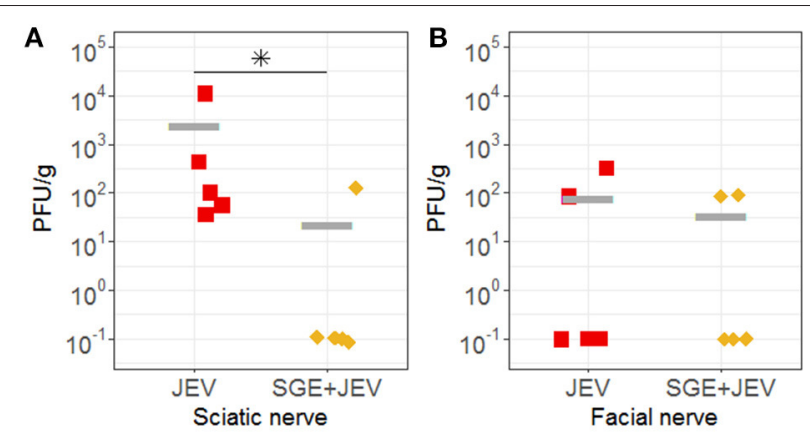

FIGURE 5 | Infectious viral titers of (A) sciatic nerve and (B) facial nerve samples collected from the infected animals. Asterisk (*) indicates the significant difference when JEV and SGE+JEV groups were compared to each other using non-parametric Mann-Whitney $U$ test.

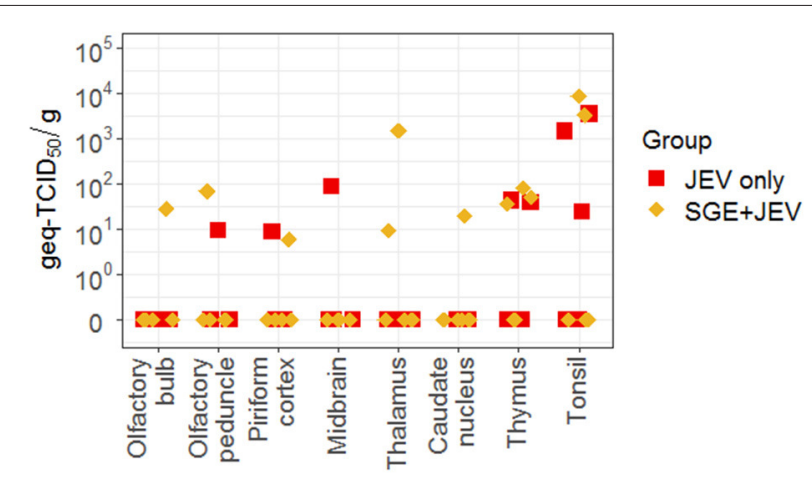

FIGURE 6 | Viral RNA detected by RT-qPCR in several central nervous and lymphoid tissues collected at 28 DPI following intradermal JEV challenge with or without SGE. DPI, days post-infection; Geq-TCID 50 , genome

equivalent-50\% tissue culture infectious dose.

pathogenesis caused by arbovirus infections. Several studies suggested that mosquito salivary components delivered through feeding or injection suppress antiviral immunity and enhance pathological outcomes in mouse models. The immunosuppressive effects of mosquito saliva include the stimulation of anti-inflammatory cytokines (34, 51, 60), polarization from a Th1 to Th2 immune response $(30,34)$, and suppression of the host innate immune responses $(29,30,33)$. However, the enhancement of pathological outcomes caused by arbovirus infections has not been consistently observed in all vertebrate species (42-44). Our study investigated the impact of mosquito SGE on the kinetics and severity of JEV infection in pigs, an amplifying host that is directly relevant to JEV transmission in nature. This model system is unique from the majority of previously published studies because it assesses the impact of mosquito salivary components on flavivirus infections in a mammalian amplifying host.

The co-injection of SGE and JEV, an established approach developed to mimic the bite of infected mosquitoes (34-37), showed that mosquito salivary components can modulate JEV infection in pigs, resulting in reduced fever and decreased nasal shedding duration. Consistent with the hypothesis that mosquito saliva suppresses the pro-inflammatory responses in the vertebrate host, the simultaneous delivery of SGE and JEV led to a low incidence and delayed onset of fever (Figure 1). This effect has also been demonstrated after mosquito bite infection of a humanized DENV mouse model, which was partially reconstituted with human immune cells to recapitulate DENV pathogenesis in humans (40). It is also consistent with the anti-inflammatory properties of $C x$. pipiens and $C x$. quinquefasciatus saliva reported in human keratinocytes $(61,62)$. Although the mechanism of how mosquito saliva caused the observed shortened duration of JEV shedding remains unclear (Figures 3A,B), lower levels of viral shedding of WNV has also been documented in two independent studies that compared chickens challenged via needle inoculation with those infected by mosquito bites $(43,63)$. Cloacal shedding of WNV was less frequently detected in chickens inoculated by infected $C x$. tritaeniorhynchus bites than chickens infected by subcutaneous injection (43). More efficient viral clearance and shortened periods of oral shedding was also reported in chicks exposed to WNV from infected $C x$. pipiens than those infected parenterally (63). However, this trend was only documented among chickens within a specific range of age (63), suggesting that reduced shedding of flaviviruses in avian hosts is also likely to be agespecific. Nevertheless, the shortened period of nasal shedding challenges the epidemiologic importance of vector-free JEV transmission (52). To date, infectious viruses and JEV genome in nasal secretions has only been detected under laboratory conditions. To the best of our knowledge, there has been no virus isolate or detection of viral genome reported in naturally infected animals, urging further investigation in understanding the degree of importance of nasal shedding of JEV among domestic pigs and whether or not it can serve as a mechanism for viral maintenance in nature.

Our results are comparable with published studies that showed that acute diseases caused by flavivirus infections can be modulated by mosquito salivary components $(35,37,40)$, but how the disease was impacted and altered was different. Feeding by infected mosquitoes or simultaneous injection of mosquito SGE with infectious viruses was implicated to enhance systemic diseases caused by flavivirus infections as observed previously, such as with inbred mouse strains challenged with WNV $(35,37)$ and humanized mice challenged with DENV serotype 2 (40). Intriguingly, the enhancement of viremia and systemic disease was not observed in our model, presumably due to the differences in the choice of vertebrate species. The seemingly contradictory outcomes may reflect the different roles of incidental and amplifying hosts in flavivirus transmission. Although a useful laboratory model, mice (Mus musculus) and other rodent species have no known role in the transmission or maintenance of flaviviruses in nature. The development of neurotropic diseases is an important pathological outcome for most mouse models after experimental challenges with flaviviruses, a hallmark that resembles the incidental hosts for JEV and other flaviviruses. On the other hand, as an amplifying host species, domestic pigs develop viremia to sustain the transmission of JEV. Although the incidence and severity of febrile illness was reduced among animals that received the intradermal injection of SGE and JEV, viremic 
titers and serum viral loads between the SGE+JEV and JEVonly groups remained comparable (Figures 2A,B). The lack of differences in viremic titers and serum viral loads indicate that the modulation of disease severity by mosquito saliva in amplification hosts potentially does not significantly affect the likelihood of transmission via the bite of infected mosquitoes. Amplifying hosts that develop viremia, but experience no apparent signs of disease, are indeed known to be advantageous for the transmission and maintenance of flaviviruses, as observed with DENV (64) and ZIKV infections in humans (65). Therefore, we speculate that the modulation of flavivirus infections by mosquito salivary components can be fundamentally different in amplifying hosts, especially mammalian species, and in incidental hosts. Additionally, consistent with the hematogenous route of neuroinvasion by JEV $(19,66-68)$, the unaltered viremia was coupled with viral burdens in the central nervous tissues that were comparable between the animals in the SGE+JEV and JEV-only groups (Figure 4). Despite the limited numbers of amplifying hosts that can be studied under laboratory conditions, understanding the differential immunomodulatory outcomes by salivary components of mosquitoes may provide an opportunity to investigate how saliva of hematophagous arthropods can affect the transmission efficiency of flaviviruses in nature. Our findings demonstrated that mosquito salivary components can modulate the kinetics of JEV infection in pigs when infectious viruses are administered at a high dosage. As the quantities of infectious viruses delivered by mosquito bites can vary significantly in natural transmission and different amount of infectious viruses present in mosquito saliva could change the outcomes of arbovirus infections (36), it is also important to determine if the modulation of arbovirus infections by mosquito saliva can be influenced by the amount of infectious viruses in an amplifying host system.

In addition to the potential variation based on the vertebrate host, the effect of mosquito saliva in the animal models may be virus-specific. Our understanding of how mosquito saliva modulates encephalitic flavivirus infections has been largely derived from WNV mouse models $(35,37,39,51,69)$. In the available WNV infection models, it has been implicated that mosquito saliva enhances the systemic infection followed by the development of more severe neuroinvasive diseases. However, our work and that of others suggest that mosquito saliva may play a different role in modulating the outcomes of JEV infections in mammalian species. For example, the co-injection of salivary glands dissected from $C x$. pipiens or Ae. albopictus and JEV had no demonstrable impact in the mortality of $\mathrm{BALB} / \mathrm{c}$ mice in comparison with intradermal inoculation of JEV alone (70). Similarly, there was no difference in the mortality rates among $\mathrm{BALB} / \mathrm{c}$ mice that receive the intradermal injection of JEVpositive saliva collected from infected mosquitoes and JEV stocks derived from cell culture (70). In contrast, high viremia and high mortality from viral encephalitis with apparent neurological signs were induced by bites from JEV-infected CX.pipiens in newborn ducklings (71), which normally do not demonstrate such clinical outcomes to JEV by needle inoculation (23). These variations in infection outcomes are most likely due to the differences in the disease pathogenesis of JEV in vertebrate species.
Differences in our and others' observations highlight the complexity of interactions among mosquitoes, JEV, and vertebrate hosts. Another factor that can contribute to the differential outcomes is the choice of mosquito species used to determine the impact of saliva or salivary components on the kinetics and severity of JEV infections. While the modulation of arbovirus infections has been reported using saliva collected from mosquito species with no or low vector competence $(38,39,51)$, the use of Culex species mosquito that are involved in natural transmission of JEV ensures the biological relevance of models developed to study the modulation of JEV infections by mosquito saliva in our and others' studies (72).

Collectively, our study demonstrates for the first time the utility of pigs to study the modulation of JEV infection by mosquito saliva. Our findings further highlight the complex and unique differences involved in mosquito-virus-host interactions. Investigating the mechanisms responsible for these differences is important to improve our understanding of the ecology and pathogenesis of arboviruses.

\section{DATA AVAILABILITY STATEMENT}

The original contributions generated for the study are included in the article/Supplementary Material, further inquiries can be directed to the corresponding author/s.

\section{ETHICS STATEMENT}

The animal study was reviewed and approved by Institutional Animal Care and Use, University Research Compliance Office, Kansas State University, Manhattan KS.

\section{AUTHOR CONTRIBUTIONS}

SP, Y-JH, SH, and DV designed the experiments. SP, Y-JH, $\mathrm{AL}, \mathrm{VA}, \mathrm{SMH}, \mathrm{DM}, \mathrm{LN}$, and $\mathrm{KB}$ conducted and executed the experimental plans. SP, Y-JH, W-WH, SH, and DV performed data analysis and drafted the manuscript. All authors contributed to the article and approved the submitted version.

\section{FUNDING}

This research was supported by the State of Kansas National Bio and Agro-Defense Facility Transition Fund. This work was supported in part by the U.S. Department of Agriculture, Agricultural Research Science, NP-103, CRIS \# 3020-32000-01400D, Cooperative Agreement 58-5430-4-021.

\section{ACKNOWLEDGMENTS}

The animal study was performed in the biosafety level 3 agriculture (BSL3-Ag) and BSL-3 laboratories at the Biosecurity Research Institute at Kansas State University. We would like to thank the Biosecurity Research Institute staff and the Comparative Medicine Group personnel at Kansas State University College of Veterinary Medicine for their technical 
assistance. Mention of trade names or commercial products in this publication is solely for the purpose of providing specific information and does not imply recommendation or endorsement by the U.S. Department of Agriculture. USDA is an equal opportunity provider and employer.

\section{REFERENCES}

1. Gould EA, Solomon T. Pathogenic flaviviruses. Lancet. (2008) 371:500-9. doi: 10.1016/S0140-6736(08)60238-X

2. Campbell GL, Hills SL, Fischer M, Jacobson JA, Hoke CH, Hombach JM, et al. Estimated global incidence of Japanese encephalitis: a systematic review. Bull World Health Organ. (2011). 89:766-74e. doi: 10.2471/BLT.10.085233

3. Schuh AJ, Ward MJ, Leigh Brown AJ, Barrett ADT. Phylogeography of Japanese encephalitis virus: genotype is associated with climate. PLoS Negl Trop Dis. (2013) 7:e2411. doi: 10.1371/journal.pntd.0002411

4. World Health Organization. Japanese Encephalitis (2019).

5. Yamada M, Nakamura K, Yoshi M, Kaku Y. Nonsuppurative encephalitis in piglets after experimental inoculation of Japanese encephalitis flavivirus isolated from pigs. Vet Pathol. (2004) 41:62-7. doi: 10.1354/vp.41-1-62

6. Yamada M, Nakamura K, Yoshii M, Kaku Y, Narita M. Brain lesions induced by experimental intranasal infection of Japanese encephalitis virus in piglets. $J$ Comp Path. (2009) 141:156-62. doi: 10.1016/j.jcpa.2009.04.006

7. Platt K, Joo H. Japanese encephalitis and West Nile viruses. In: Straw BE, Zimmerman JJ, D’allaire S, Taylor DJ, editors. Diseases of Swine. Ames, IA: Blackwell Publishing (2006). p. 359-64.

8. Shimizu T, Kawakami Y, Fukuhara S, Matumoto M. Experimental stillbirth in pregnant swine infected with Japanese Encephalitis virus. Jpn J Exp Med. (1954) 24:363-75.

9. Desingu PA, Ray PK, Patel BH, Singh R, Singh RK, Saikumar G. Pathogenic and genotypic characterization of a japanese encephalitis virus isolate associated with reproductive failure in an Indian pig herd. PLoS ONE. (2016) 11:e0147611. doi: 10.1371/journal.pone.0147611

10. Ravanini P, Huhtamo E, Ilaria V, Crobu M, Nicosia A, Servino L, et al. Japanese encephalitis virus RNA detected in Culex pipiens mosquitoes in Italy. Euro Surveill. (2012). 17:20221. doi: 10.2807/ese.17.28.20221-en

11. Preziuso S, Mari S, Mariotti F, Rossi G. Detection of Japanese Encephalitis Virus in bone marrow of healthy young wild birds collected in 19972000 in Central Italy. Zoonoses Public Health. (2018) 65::798-804. doi: 10.1111/zph.12501

12. Simon-Loriere E, Faye O, Prot M, Casademont I, Fall G, FernandezGarcia MD, et al. Autochthonous Japanese Encephalitis with Yellow Fever coinfection in Africa. $N$ Engl J Med. (2017) 376:1483-5. doi: 10.1056/NEJMc1701600

13. Ghosh D, Basu A. Japanese encephalitis-a pathological and clinical perspective. PLoS Negl Trop Dis. (2009) 3:e437. doi: 10.1371/journal.pntd.0000437

14. Tsuchiya T. Intrabulbar inoculation of Japanese encephalitis virus to mice. Kurume Med J. (1968) 15:43-50. doi: 10.2739/kurumemedj.15.43

15. Mathur A, Arora KL, Chaturvedi UC. Congenital infection of mice with Japanese Encephalitis virus. Infect Immun. (1981) 34:26-9. doi: 10.1128/iai.34.1.26-29.1981

16. Li Y, Counor D, Lu P, Duong V, Yu Y, Deubel V. Protective immunity to Japanese encephalitis virus associated with anti-NS1 antibodies in a mouse model. Virol J. (2012) 9:135. doi: 10.1186/1743-422X-9-135

17. Cao L, Fu S, Gao X, Li M, Cui S, Li X, et al. Low protective efficacy of the current Japanese Encephalitis Vaccine against the emerging genotype 5 Japanese Encephalitis Virus. PLoS Negl Trop Dis. (2016) 10:e0004686. doi: 10.1371/journal.pntd.0004686

18. Myint KS, Raengsakulrach B, Young GD, Gettayacamin M, Ferguson LM, Innis BL, et al. Production of lethal infection that resembles fatal human disease by intranasal inoculation of macaques with Japanese encephalitis virus. Am J Trop Med Hyg. (1999) 60:338-42. doi: 10.4269/ajtmh.1999. 60.338

19. Myint KSA, Kipar A, Jarman RG, Gibbons RV, Perng GC, Flanagan B, et al. Neuropathogenesis of Japanese Encephalitis in a primate model. PLoS Negl Trop Dis. (2014) 8:e2980. doi: 10.1371/journal.pntd.0002980

\section{SUPPLEMENTARY MATERIAL}

The Supplementary Material for this article can be found online at: https://www.frontiersin.org/articles/10.3389/fviro. 2021.724016/full\#supplementary-material

20. Raengsakulrach B, Nisalak A, Gettayacamin M, Thirawuth V, Young GD, Myint KS, et al. An intranasal challenge model for testing Japanese encephalitis vaccines in rhesus monkeys. Am J Trop Med Hyg. (1999) 60:32937. doi: 10.4269/ajtmh.1999.60.329

21. Cleton NB, Bosco-Lauth A, Page MJ, Bowen RA. Age-related susceptibility to Japanese encephalitis virus in domestic ducklings and chicks. Am J Trop Med Hyg. (2014) 90:242-6. doi: 10.4269/ajtmh.13-0161

22. Xiao C, Li C, Di D, Cappelle J, Liu L, Wang X, et al. Differential replication efficiencies between Japanese encephalitis virus genotype I and III in avian cultured cells and young domestic ducklings. PLoS Negl Trop Dis. (2018) 12:e0007046. doi: 10.1371/journal.pntd.0007046

23. Xiao C, Wang X, Cui G, Pang L, Xu J, Li C, et al. Possible pathogenicity of Japanese encephalitis virus in newly hatched domestic ducklings. Vet Microbiol. (2018) 227:8-11. doi: 10.1016/j.vetmic.2018.10.016

24. Williams DT, Daniels PW, Lunt RA, Wang L-F, Newberry KM, Mackenzie JS. Experimental infections of pigs with Japanese encephalitis virus and closely related Australian flaviviruses. Am J Trop Med Hyg. (2001) 65:379-87. doi: 10.4269/ajtmh.2001.65.379

25. Ricklin ME, Garcia-Nicolas O, Brechbuhl D, Python S, Zumkehr B, Posthaus $\mathrm{H}$, et al. Japanese encephalitis virus tropism in experimentally infected pigs. Vet Res. (2016) 47:34. doi: 10.1186/s13567-016-0319-Z

26. Park SL, Huang Y-JS, Lyons AC, Ayers VB, Hettenbach SM, Mcvey DS, et al. North American domestic pigs are susceptible to experimental infection with Japanese encephalitis virus. Sci Rep. (2018) 8:7951. doi: 10.1038/s41598-018-26208-8

27. Young CL, Lyons AC, Hsu W-W, Vanlandingham DL, Park SL, Bilyeu AN, et al. Protection of swine by potent neutralizing anti-Japanese encephalitis virus monoclonal antibodies derived from vaccination. Antiviral Res. (2020) 174:104675. doi: 10.1016/j.antiviral.2019.104675

28. Osorio JE, Godsey MS, Defoliart GR, Yuill TM. La Crosse viremias in whitetailed deer and chipmunks exposed by injection or mosquito bite. Am J Trop Med Hyg. (1996) 54:338-42. doi: 10.4269/ajtmh.1996.54.338

29. Schneider BS, Higgs S. The enhancement of arbovirus transmission and disease by mosquito saliva is associated with modulation of the host immune response. Trans $R$ Soc Trop Med Hyg. (2008) 102:400-8. doi: 10.1016/j.trstmh.2008.01.024

30. Thangamani S, Higgs S, Ziegler S, Vanlandingham D, Tesh R, Wikel S. Host immune response to mosquito-transmitted chikungunya virus differs from that elicited by needle inoculated virus. PLOS ONE. (2010) 5:e12137. doi: 10.1371/journal.pone.0012137

31. Conway MJ, Colpitts TM, Fikrig E. Role of the vector in Arbovirus transmission. Annu Rev Virol. (2014) 1:71-88. doi: 10.1146/annurev-virology-031413-085513

32. Pingen M, Bryden SR, Pondeville E, Schnettler E, Kohl A, Merits A, et al. Host inflammatory response to mosquito bites enhances the severity of arbovirus infection. Immunity. (2016) 44:1455-69. doi: 10.1016/j.immuni.2016.06.002

33. Fong S-W, Kini RM, Ng LFP. Mosquito saliva reshapes alphavirus infection and immunopathogenesis. J Virol. (2018) 92:e01004-17. doi: 10.1128/JVI.01004-17

34. Schneider BS, Soong L, Zeidner NS, Higgs S. Aedes aegypti salivary gland extracts modulate anti-viral and TH1/TH2 cytokine responses to sindbis virus infection. Viral Immunol. (2004) 17:565-73. doi: 10.1089/vim.2004.17.565

35. Styer LM, Lim P-Y, Louie KL, Albright RG, Kramer LD, Bernard KA. Mosquito saliva causes enhancement of West Nile virus infection in mice. $J$ Virol. (2011) 85:1517-27. doi: 10.1128/JVI.01112-10

36. Le Coupanec A, Babin D, Fiette L, Jouvion G, Ave P, Misse D, et al. Aedes mosquito saliva modulates Rift Valley fever virus pathogenicity. PLoS Negl Trop Dis. (2013) 7:e2237. doi: 10.1371/journal.pntd.0002237

37. Moser LA, Lim PY, Styer LM, Kramer LD, Bernard KA. Parameters of mosquito-enhanced West Nile virus infection. J Virol. (2016) 90:292-9. doi: 10.1128/JVI.02280-15 
38. Edwards JF, Higgs S, Beaty BJ. Mosquito feeding-induced enhancement of Cache Valley Virus (Bunyaviridae) infection in mice. J Med Entomol. (1998) 35:261-5. doi: 10.1093/jmedent/35.3.261

39. Schneider BS, Soong L, Girard YA, Campbell G, Mason P, Higgs S. Potentiation of West Nile encephalitis by mosquito feeding. Viral Immunol. (2006) 19:74-82. doi: 10.1089/vim.2006.19.74

40. Cox J, Mota J, Sukupolvi-Petty S, Diamond MS, Rico-Hesse R. Mosquito bite delivery of dengue virus enhances immunogenicity and pathogenesis in humanized mice. J Virol. (2012) 86:7637-49. doi: 10.1128/JVI. 00534-12

41. Conway MJ, Watson AM, Colpitts TM, Dragovic SM, Li Z, Wang P, et al. Mosquito saliva serine protease enhances dissemination of dengue virus into the mammalian host. J Virol. (2014) 88:164-75. doi: 10.1128/JVI.02235-13

42. Sbrana E, Tonry JH, Xiao SY, Da Rosa AP, Higgs S, Tesh RB. Oral transmission of West Nile virus in a hamster model. Am J Trop Med Hyg. (2005) 72:325-9. doi: 10.4269/ajtmh.2005.72.325

43. Langevin SA, Bunning M, Davis B, Komar N. Experimental infection of chickens as candidate sentinels for West Nile virus. Emerg Infect Dis. (2001) 7:726-9. doi: 10.3201/eid0704.017422

44. Reisen WK, Chiles RE, Kramer LD, Martinez VM, Eldridge BF. Method of infection does not alter response of chicks and house finches to western equine encephalomyelitis and St. Louis encephalitis viruses. J Med Entomol. (2000) 37:250-8. doi: 10.1603/0022-2585-37.2.250

45. Huang YJS, Hettenbach SM, Park SL, Higgs S, Barrett ADT, Hsu WW, et al. Differential infectivities among different Japanese Encephalitis Virus genotypes in Culex quinquefasciatus mosquitoes. PLoS Negl Trop Dis. (2016) 10:e0005038. doi: 10.1371/journal.pntd.0005038

46. Reed LJ, Muench H. A simple method of estimating fifty per cent endpoints. Am J Epidemiol. (1938) 27:493-7. doi: 10.1093/oxfordjournals.aje. a118408

47. Higgs S, Vanlandingham DL, Klingler KA, Mcelroy KL, Mcgee CE, Harrington $\mathrm{L}$, et al. Growth characteristics of ChimeriVax-Den vaccine viruses in Aedes aegypti and Aedes albopictus from Thailand. Am J Trop Med Hyg. (2006) 75:986-93. doi: 10.4269/ajtmh.2006.75.986

48. Huang YJ, Ayers VB, Lyons AC, Unlu I, Alto BW, Cohnstaedt LW, et al. Culex species mosquitoes and Zika Virus. Vector Borne Zoonotic Dis. (2016) 16:673-6. doi: 10.1089/vbz.2016.2058

49. Ayers VB, Huang YS, Lyons AC, Park SL, Higgs S, Dunlop JI, et al. Culex tarsalis is a competent vector species for Cache Valley virus. Parasit Vectors. (2018) 11:519. doi: 10.1186/s13071-018-3103-2

50. Cook CL, Huang YS, Lyons AC, Alto BW, Unlu I, Higgs S, et al. North American Culex pipiens and Culex quinquefasciatus are competent vectors for Usutu virus. PLoS Negl Trop Dis. (2018) 12:e0006732. doi: 10.1371/journal.pntd.0006732

51. Schneider BS, Soong L, Coffey LL, Stevenson HL, McGee CE, Higgs S. Aedes aegypti saliva alters leukocyte recruitment and cytokine signaling by antigenpresenting cells during West Nile virus infection. PLoS ONE. (2010) 5:e11704. doi: 10.1371/journal.pone.0011704

52. Ricklin ME, Garcia-Nicolas O, Brechbuhl D, Python S, Zumkehr B, Nougairede A, et al. Vector-free transmission and persistence of Japanese Encephalitis virus in pigs. Nat Commun. (2016) 7:10832. doi: $10.1038 /$ ncomms 10832

53. Baer A, Kehn-Hall K. Viral concentration determination through plaque assays: using traditional and novel overlay systems. J Vis Exp. (2014) 2014:e52065. doi: 10.3791/52065

54. Pyke AT, Smith IL, Van Den Hurk AF, Northill JA, Chuan TF, Westacott AJ, et al. Detection of Australasian Flavivirus encephalitic viruses using rapid fluorogenic TaqMan RT-PCR assays. J Virol Methods. (2004) 117:161-7. doi: 10.1016/j.jviromet.2004.01.007

55. Lyons AC, Huang YS, Park SL, Ayers VB, Hettenbach SM, Higgs S, et al. Shedding of Japanese Encephalitis Virus in oral fluid of infected swine. Vector Borne Zoonotic Dis. (2018) 18:469-74. doi: 10.1089/vbz.2018.2283

56. Roehrig J, Hombach J, Barrett A. Guidelines for plaque reduction neutralization testing of human antibodies to dengue viruses. Viral Immunol. (2008) 21:123-32. doi: 10.1089/vim.2008.0007

57. García-Nicolás O, Ricklin ME, Liniger M, Vielle NJ, Python S, Souque P, et al. A Japanese Encephalitis Virus vaccine inducing antibodies strongly enhancing in vitro infection is protective in pigs. Viruses. (2017) 9:124. doi: $10.3390 / \mathrm{v} 9050124$
58. Kodama K, Sasaki N, Inoue YK. Studies of live attenuated Japanese Encephalitis vaccine in swine. J Immunol. (1968) 100:194-200.

59. Gulati BR, Singha H, Singh BK, Virmani N, Khurana SK, Singh RK. Serosurveillance for Japanese encephalitis virus infection among equines in India. J Vet Sci. (2011) 12:341-5. doi: 10.4142/jvs.2011.12.4.341

60. Vogt MB, Lahon A, Arya RP, Kneubehl AR, Spencer Clinton JL, Paust S, et al. Mosquito saliva alone has profound effects on the human immune system. PLoS Negl Trop Dis. (2018) 12:e0006439. doi: 10.1371/journal.pntd.0006439

61. Zeidner NS, Higgs S, Happ CM, Beaty BJ, Miller BR. Mosquito feeding modulates Th1 and Th2 cytokines in flavivirus susceptible mice: an effect mimicked by injection of sialokinins, but not demonstrated in flavivirus resistant mice. Parasite Immunol. (1999) 21:35-44. doi: 10.1046/j.1365-3024.1999.00199.x

62. Garcia M, Alout H, Diop F, Damour A, Bengue M, Weill M, et al. Innate immune response of primary human keratinocytes to West Nile Virus infection and its modulation by mosquito saliva. Front Cell Infect Microbiol. (2018) 8:387. doi: 10.3389/fcimb.2018.00387

63. Styer LM, Bernard KA, Kramer LD. Enhanced early West Nile virus infection in young chickens infected by mosquito bite: effect of viral dose. Am J Trop Med Hyg. (2006) 75:337-45. doi: 10.4269/ajtmh.2006.75.337

64. Ten Bosch QA, Clapham HE, Lambrechts L, Duong V, Buchy $\mathrm{P}$, Althouse BM, et al. Contributions from the silent majority dominate dengue virus transmission. PLoS Pathog. (2018) 14:e1006965. doi: 10.1371/journal.ppat.1006965

65. Moghadas SM, Shoukat A, Espindola AL, Pereira RS, Abdirizak F, Laskowski $\mathrm{M}$, et al. Asymptomatic transmission and the dynamics of Zika infection. Sci Rep. (2017) 7:5829. doi: 10.1038/s41598-017-05013-9

66. Monath TP, Cropp CB, Harrison AK. Mode of entry of a neurotropic arbovirus into the central nervous system. Reinvestigation of an old controversy. Lab Invest. (1983) 48:399-410.

67. Mathur A, Khanna N, Chaturvedi U. Breakdown of blood-brain barrier by virus-induced cytokine during Japanese encephalitis virus infection. Int J Exp Path. (1992) 73:603-11.

68. Nagata N, Iwata-Yoshikawa N, Hayasaka D, Sato Y, Kojima A, Kariwa H, et al. The pathogenesis of 3 neurotropic flaviviruses in a mouse model depends on the route of neuroinvasion after Viremia. J Neuropathol Exp Neurol. (2015) 74:250-60. doi: 10.1097/NEN.0000000000000166

69. Schneider BS, Mcgee CE, Jordan JM, Stevenson HL, Soong L, Higgs S. Prior exposure to uninfected mosquitoes enhances mortality in naturally-transmitted West Nile virus infection. PLoS ONE. (2007) 2:e1171. doi: 10.1371/journal.pone.0001171

70. De Wispelaere M, Desprès P, Choumet V. European Aedes albopictus and Culex pipiens are competent vectors for Japanese Encephalitis virus. PLoS Negl Trop Dis. (2017) 11:e0005294. doi: 10.1371/journal.pntd.0005294

71. Di D, Li C, Zhang J, Hameed M, Wang X, Xia Q, et al. Experimental infection of newly hatched domestic ducklings via Japanese Encephalitis virus-infected mosquitoes. Pathogens. (2020) 9:371. doi: 10.3390/pathogens9050371

72. Weng MH, Lien JC, Wang YM, Lin CC, Lin HC, Chin C. Isolation of Japanese encephalitis virus from mosquitoes collected in Northern Taiwan between 1995 and 1996. J Microbiol Immunol Infect. (1999) 32:9-13.

Conflict of Interest: The authors declare that the research was conducted in the absence of any commercial or financial relationships that could be construed as a potential conflict of interest.

Publisher's Note: All claims expressed in this article are solely those of the authors and do not necessarily represent those of their affiliated organizations, or those of the publisher, the editors and the reviewers. Any product that may be evaluated in this article, or claim that may be made by its manufacturer, is not guaranteed or endorsed by the publisher.

Copyright (C) 2021 Park, Huang, Lyons, Ayers, Hettenbach, McVey, Noronha, Burton, Hsu, Higgs and Vanlandingham. This is an open-access article distributed under the terms of the Creative Commons Attribution License (CC BY). The use, distribution or reproduction in other forums is permitted, provided the original author(s) and the copyright owner(s) are credited and that the original publication in this journal is cited, in accordance with accepted academic practice. No use, distribution or reproduction is permitted which does not comply with these terms. 